\title{
MDM2 RNA binding is blocked by novel monoclonal antibody h-MDM2-F4-14
}

\author{
JOHN J. ANDERSON ${ }^{1}$, CHRISTINE CHALLEN ${ }^{2}$, HELEN ATKINS ${ }^{2}$, \\ R. SUAEYUN ${ }^{2}$, STEPHEN CROSIER ${ }^{1}$ and JOHN LUNEC ${ }^{2}$ \\ ${ }^{1}$ Department of Academic Haematology, The University of Newcastle Upon Tyne, NE1 4LP; \\ ${ }^{2}$ The Northern Institute For Cancer Research, The University of Newcastle Upon Tyne, NE2 4HH, UK
}

Received February 23, 2007; Accepted May 29, 2007

\begin{abstract}
Amplification of MDM2 has been described in a variety of human cancers. Prognostic studies have revealed that abnormal MDM2 expression correlates with poor prognosis. Many of the consequences of MDM2/p53 interactions have been investigated, and MDM2-p53 dependent events characterized. In contrast, understanding of MDM2p53 independent activities is comparatively in it's infancy amongst these the ability of MDM2 to bind RNA. However, although the significance of this activity has been the subject of some speculation, the precise role and impact of this function upon cell replication or apoptosis has yet to be fully defined. These studies have been obstructed by a lack of specific reagents able to interfere with this reaction. As a prelude to further exploring the significance of MDM2 RNA binding we report the inhibition of MDM2 RNA binding activity by newly produced MDM2 monoclonal antibodies anti-h-MDM2 F4-14 and F2-2. A variety of MDM2 specific antibodies have been produced and applied in research without complete knowledge of their reactivity profiles, but in the face of the growing number of MDM2 RNA isoforms, the results of such studies can be difficult to interpret. Each of the RNA binding inhibitory antibodies produced in this study was found to be reactive with full-length MDM2 protein expressed in tumor cell lysates, transfected NIH3T3 cell lysates and via eukaryotic cell free rabbit reticulocyte in vitro translation. Antibody F4-14, the most potently inhibitory antibody, reacts strongly with the full length MDM2 together with protein isoforms A, B, C and D. In contrast, antibody F2-2 reacts only with full-length MDM2 protein. The ability of h-MDM2-F4-14
\end{abstract}

Correspondence to: Dr J.J. Anderson, Department of Academic Haematology, The University of Newcastle Upon Tyne, NE2 4HH, UK

E-mail: j.j.anderson@ncl.ac.uk

Dr C. Challen, The Northern Institute for Cancer Research, Paul O'Gorman Building, The University of Newcastle Upon Tyne, NE2 4HH, UK

Key words: MDM2, RNA binding, therapeutic targets and to a lesser extent F2-2 to inhibit RNA binding presents the possibility of modulating human MDM2s ability to bind RNA, compromise this function and present opportunities to investigate in more detail the biological significance of this activity.

\section{Introduction}

The MDM2 oncogene was originally cloned from a spontaneously transformed BALB/c 3 T3 mouse cell line where it was found to be amplified and over-expressed $(1,2)$. The human homologue is located at chromosomal position 12q13-14 and shares $80.3 \%$ identity in coding sequence with murine mdm2 (3). The human MDM2 gene itself encodes a 491 amino acid (a.a.) protein that migrates with an apparent molecular weight of $90 \mathrm{kDa}$ in PAGE (projected weight $56 \mathrm{kDa}$ ). Deletion and sequence analysis have shown that the MDM2 nuclear phosphoprotein contains a p53 binding site in the amino terminal region (codons 19-102), a putative nuclear localization signal (codons 181-185), an acidic activation domain (codons 223-274), central zinc finger motifs (codons 305-322) and a carboxy-terminal RING finger zinc binding motif (codons 438-478) (4,5) suggesting nucleic acid binding and/or transcription factor functions. The tumor suppressor gene product $\mathrm{p} 53$, can induce the expression of MDM2 via transactivation of the p53 dependent promoter of the MDM2 gene, thereby activating an auto-regulatory feedback loop (6-8), the terminal event of which is the binding of MDM2 to p53 that effectively targets p53, at low levels for export from the nucleus and at higher levels for ubiquitin dependent degradation (9). Although as an E3 ubiquitin ligase its activity is by no means confined to p53 but also encompases other key cell cycle associated proteins including pRB as well as histones H2A and H2B (10). However, the regulatory functions of MDM2 are not confined to its role as a ubiquitin ligase. As the breadth of MDM2 focused research increasingly expands, it is becoming correspondingly clearer that human MDM2 is truly a pluripotent molecule, whose action upon and interaction with other molecules modulate cell proliferation, growth signal response pathways, and apoptosis (11). While many MDM2 protein interactions have been highlighted, it has also been shown that it possesses nucleic acid binding activity, this first being recognized by the demonstration that bacculovirus expressed MDM2 
binds RNA specifically through the RING finger domain of the protein and that this binding is abrogated when a single amino acid substitution is made in the RING finger domain (12).We have confirmed this observation using a bacterially expressed MDM2 protein and have also gained evidence of atypical DNA binding activity (C. Challen personal communication). Amplification of the MDM2 gene has been described in a number of human cancers, and a high frequency of occurrence has been reported in a number of soft tissue sarcomas as well as osteosarcomas and gliomas $(13,14)$. Over-expression of the MDM2 protein may arise from gene amplification, but may also occur by enhanced transcription or translation as documented in lymphoid and myeloid leukaemias $(14,15)$. Increased MDM2 expression via whichever means may precipitate inactivation of p53 function and consequently inhibit cellular responses to DNA damage or cellular stress i.e. failure to induce p53 'responsive genes'. Furthermore, alternately spliced MDM2 transcripts have been identified in vivo that can be translated from expression constructs in vitro and display enhanced transforming properties (16). This is particularly true of the shortest transcripts, lacking the p53-binding site, which display the greatest transforming activity. However, the precise role of the many alternatively spliced transcripts derived from the h-MDM2 gene has yet to be fully elucidated, this task being compounded by the diversity of these products which now exceeds 40 in number (17).

Clinically orientated studies have revealed that evaluation of MDM2 expression may be of prognostic significance, although this remains contentious as the value of MDM2 as a marker of poor prognosis is somewhat tumor dependent (18). Although over-expression of MDM2 could potentially enhance the effect of functional p53 disturbance by augmenting proliferation, MDM2 and p53 abnormalities within the same tumor are uncommon (13). However, the occurrence of abnormal MDM2 expression and p53 mutations and/or combined over-expression has been correlated with poor patient prognosis and reduced survival. Furthermore, these abnormalities have also been correlated with pathophysiological markers of tumor aggression including factors such as metastasis, advanced tumor stage, poor differentiation and high grade. Such correlations have been described in a variety of tumors including soft tissue sarcomas and carcinoma of the bronchus, breast and urinary bladder (19-22). However, the complexity and diversity of MDM2 interactions and their relative physiological importance in cells of different lineages determine the predictive value of MDM2 over-expression. For example whilst MDM2 over expression is a predictive marker of poor prognosis soft tissue sarcomas, subpopulations of gliomas, and pediatric acute lymphoblastic leukemia the reverse appears to be the case in melanoma, $\mathrm{ER} \alpha^{+}$breast carcinoma and non-small cell lung cancer (18). A variety of different methodologies and different antibodies have been employed in the various prognostic studies that have in the most relied upon immunohistochemical methods. In such studies it may be difficult to compare results, as antibody reactivity may in instances be different with respect to the epitopes and protein isoforms with which individual reagents react. Thus, comparative analysis of these findings can prove difficult.
Understanding the underlying complexity of MDM2 interactions in their appropriate cellular context will provide the means of manipulating those p53 dependent or independent pathways that may modulate proliferation, survival or resposnsiveness to stimuli. Current understanding of these processes are opening up opportunities for new targeted therapies; such as synthesis of 'Syc' 7 a molecule targeting p53 responsive genes promoting apoptosis in instances where p53 function may be blocked by MDM2; or the production of cis-imidazoline analogues which act as inhibitors/competitors of the MDM2/p53 complex formation itself. To date, little research has focused on the effect of modulating MDM2: RNA binding as such studies have been limited by the availability of appropriate reagents. In the current study we report the derivation and ability of two monoclonal antibodies to abolish interaction of MDM2 with its defined RNA consensus binding sequence opening the possibility to explore this little studied effect both in vivo and in vitro. Furthermore, we confirm the reactivity of these antibodies both with native MDM2 FL protein in tumor cell lysates and characterize their reactivity with a number of the principle MDM2 isoforms expressed both in cell free in vitro translation and cell transfection studies.

\section{Materials and methods}

Cloning of MDM2 sequences from total cellular RNA. Total cellular RNA was extracted from sub-confluent monolayers of $\mathrm{CaCO}_{2}$-colonic carcinoma cells using conventional manufacturer defined RNAzol based extraction protocols (Biogenesis Ltd., UK). A full-length cDNA clone of MDM2 was generated by RT-PCR employing a specific primer pair, facilitating amplification of the entire coding sequence: (sense primer: 5'-CGCGAAAACCCCGGGCAGGCAAAG TGCA-3', antisense primer: 5'-CTCTTATAGACAGGTCA ACTAG-3'). The primers designed to amplify the full length product incorporated BamH1 restriction sites and appropriate additional bases to ensure maintenance of reading frame on sub-cloning into the expression vector. Conventional AMVRT-PCR protocols were applied throughout (Promega Ltd., UK). The PCR product was resolved on $0.8 \% \mathrm{w} / \mathrm{v}$ agaroseTBE gels, then gel purified and A: T cloned into the general purpose pUC57T plasmid utilising T4 DNA ligase in the presence of PEG4000 and BSA. The resulting construct was then transformed into Novablue E. coli (DE3) and transformants identified by growth on 2YT-TAXI (tetracycline, ampicillin, X-gal and IPTG) selective agar plates. PCR and restriction mapping confirmed the identity of the insert in constructs from the transformants and finally dideoxygenic sequencing validated the fidelity of selected inserts.

Generation of MDM2 expression constructs. Full-length MDM2 inserts were excised from pUC57T using BamH1 and re-ligated in frame into the complementary site within the multiple cloning site of the pET15b (Novagen) expression vector. The resulting product was then transformed into competent T7pol- E. coli Novablue (DE3) bacteria. Transformants were selected initially by growth on TC (tetracycline and carbenicillin)-2YT agar plates. Subsequently transformants were screened by PCR, which confirmed insert 
identity, size and orientation. Stable clones, which retained plasmid, were expanded and recombinant plasmid DNA was purified using P3-DNA plasmid mini-prep-kits (Helena Biosciences Ltd., UK) adhering to the manufacturer's protocols. After further confirmation of insert fidelity by automated DNA sequencing, several constructs were retransformed into $\mathrm{T} \mathrm{pol}^{+}$E. coli BL21 (pLysS) and protein expression induced in small-scale broth culture by IPTG. Cells were harvested pre- and post-induction and the yield of protein and the solubility of the product expressed by various clones was determined employing a combination of differential centrifugation and PAGE electrophoresis of bacterial cell lysates. The final selection of the clone to be employed to produce the recombinant protein was based upon determination of optimum yields of protein. Recombinant Nterminal-poly-histidine (6X-His) tagged MDM2 -FL (fulllength) protein was induced in large-scale spinner cultures and cells harvested 4 to 6 -h post IPTG induction. Initial experiments demonstrated that the recombinant protein resided in the insoluble fraction of the cells localized in the cytoplasmic granules. The protein was recovered by initially purifying cytoplasmic granules from $E$. coli by differential centrifugation and subsequently solubilising the protein in $1 \mathrm{mM}$ imidazole, $0.12 \mathrm{M} \mathrm{NaCl}, 10 \mathrm{mM}$ Tris- $\mathrm{HCl} \mathrm{pH} 7.9$ containing $8 \mathrm{M}$ urea in the presence of $2 \mathrm{mM} 2$-mercaptoethanol $(2 \mathrm{ME})$. The protein was then refolded by dialysis against Tris- $\mathrm{HCl} /$ urea solutions $(\mathrm{pH} 8.0)$ in which the concentration of urea was progressively reduced in serial increments from 6 to $0 \mathrm{M}$. Throughout, $2 \mathrm{ME}$ concentrations were maintained until urea was excluded from the dialysate at which point $2 \mathrm{ME}$ concentrations were reduced to zero over $96 \mathrm{~h}$. This procedure produced a protein preparation that before nickel column affinity chromatography (standard manufacturer's procedures), contained greater than $95 \%$ recombinant MDM2. Aliquots of this crude recombinant protein were adjusted to $500-1000 \mu \mathrm{g} / \mathrm{ml}$ and stored at $-20^{\circ} \mathrm{C}$ until required.

Chromatographic purification of MDM2 poly-histidine tagged protein. The dialysate from above was purified employing $2.5 \mathrm{ml}$ His-Bind-Resin columns. These columns were charged with $\mathrm{Ni}^{2+}$ ions and equilibrated with bind buffer (1 mM imidazole, $0.12 \mathrm{M} \mathrm{NaCl}, 10 \mathrm{mM}$ Tris- $\mathrm{HCl} \mathrm{pH}$ 7.9). The dialysate, diluted in bind buffer was loaded onto the column at a flow rate of approximately $2 \mathrm{ml} / \mathrm{min}$. Unbound protein was then washed off the column with buffer containing $15 \mathrm{mM}$ imidazole, $0.25 \mathrm{M} \mathrm{NaCl}, 0.125 \mathrm{M}$ Tris- $\mathrm{HCl} \mathrm{pH}$ 7.9. immobilized protein was then eluted from the column in a solution of $4.9 \mathrm{mM}$ imidazole, $0.25 \mathrm{M} \mathrm{NaCl}$ and $0.25 \mathrm{M}$ Tris$\mathrm{HCl} \mathrm{pH}$ 7.9. Subsequently, $0.5-1.0 \mathrm{ml}$ fractions of eluate were collected from the column which was then cleaned and regenerated according to manufacturer's protocols. The protein concentration, of each fraction of eluate was determined by Bradford assay, and its content analyzed by SDS-PAGE. Fractions containing pure recombinant MDM2 protein were pooled; the protein concentration of the pool determined and then aliquots were stored at $-80^{\circ} \mathrm{C}$ until required.

Production of MDM2-specific monoclonal antibodies. $\mathrm{BALB} / \mathrm{c}$ mice were immunized with FL recombinant MDM2 protein according to previously described immunization schedules (23). In this instance optimum serum antibody responses were produced when the primary and secondary subcutaneous bolus of antigen was given as water in oil in water (WOW) emulsion, in 'Freund's incomplete adjuvant'. All further booster injections were administered intraperitoneally, diluted in physiological saline. A final intravenous booster of 50-100 $\mu \mathrm{g}$ of antigen was given to each animal $72 \mathrm{~h}$ prior to fusion. Throughout the entire immunization schedule, serum antibody induction was monitored by enzyme-linked immunosorbent-assay (ELISA). Species specific (goat anti-mouse or goat anti-rabbit) alkaline phosphatase conjugated antibodies (KPL, Ltd.) $(1 / 5,000)$ were used as detector anti-bodies throughout all experiments while p-nitro-phenol-phosphate (PNP) in alkaline phosphatase buffer served as the chromogenic substrate. Reactions were halted with sodium hydroxide $(3 \mathrm{M})$ and binding of the conjugate was quantified spectrophotometrically by measuring absorbance at $590 \mathrm{~nm}$.

Immune splenocytes from responsive mice were fused with NS-1/Ag-3 myeloma cells using established protocols (23). The resulting hybridoma cells, suspended in selective HAT-RPMI-1640-20\% FCS medium supplemented with $10 \% \mathrm{v} / \mathrm{v}$ Condimed (Boehringer Mannheim), were plated in $200 \mu \mathrm{l}$ volumes into 96-well FB-Falcon plates which were incubated at $37^{\circ} \mathrm{C}$ for $7-10$ days. After this time each culture was screened for specific antibody production. Primary screening was performed by ELISA, employing homologous recombinant FL MDM2 His-tagged protein $\left(5 \times 10^{-8} \mathrm{M}\right)$ as capture antigen. An MDM2 GST-N terminal deletion construct-protein product was also employed in secondary rounds of screening. Individual colonies were picked from antibody positive wells and transferred to 48-well plates. Subsequent rounds of limiting dilution, re-enforced by selective screening by ELISA and Western blotting, helped ensure the specificity of the expanded antibody clones. From the primary panel of hybridomas produced, a number of clones were selected for further characterization both by immunohistochemistry, Western blotting and finally in mobility shiftretardation assays. Ultimately two hybridomas were selected: these secreted monoclonal antibodies h-MDM2-F2-2 and h-MDM2-F4-14.

Purification of monoclonal antibodies using protein $G$ column chromatography. F2-2 (IgG1) (1.5 1) and F4-14 (IgG1) hybridoma culture supernatants were each fractionated by column chromatography using Prosep-Protein A/G columns in accordance with the manufacturer's protocol. Aliquots (12-16 ml) of purified F2-2 and F4-14 monoclonal antibody eluates were obtained from this starting material. The protein content of each pooled eluate was determined by Bradford assay $(18 \mathrm{mg} / \mathrm{ml}$ and $13.4 \mathrm{mg} / \mathrm{ml})$ and aliquots stored at $-80^{\circ} \mathrm{C}$.

Western blotting. The MDM2 specificity of individual antibodies was confirmed by Western blotting. Initially $\mathrm{CaCo}_{2}$ cell lysates, previously found to contain elevated levels of MDM2 were employed in preliminary experiments. In later studies, FL-MDM2 recombinant protein, in vitro translated/ transcribed spliced $M D M 2$ transcripts, or lysates of induced (as well as control uninduced cells) MDM2 construct trans- 
fected cell lysates were resolved on $10 \% \mathrm{~T} / 2.6 \% \mathrm{C}$ SDS PAGE resolving gels over-layered with $4 \% \mathrm{~T} / 2.6 \% \mathrm{C}$ stacking gels. Products were transferred to BA65 nitrocellulose membranes (Schleicher \& Schuell Ltd.) and antibody reactivity with transferred products was visualized employing mouse-immunoglobulin-specific horseradish peroxidase (HRP) conjugated secondary antibodies $(1 / 5,000-1 / 10,000)$ using conventional ECL ${ }^{\mathrm{TM}}$ methodology (National Diagnostics Ltd., USA) in conjunction with Fuji-RX photo-graphic film.

Immunohistochemistry (IHC). Blocks of normal and tumor tissue, which were previously fixed in neutral buffered formalin and embedded in paraffin wax in accordance with conventional protocols, were selected from archives for use in this study. Tissue sections $(5 \mu \mathrm{m})$, cut from these blocks were mounted on amino-propyl-tri-ethoxysilane (APES) coated microscope slides and then de-waxed, re-hydrated, subjected to antigen retrieval (citrate phosphate buffer $\mathrm{pH} 5.6$ plus microwave treatment) and subsequently reacted with MDM2 specific monoclonal antibodies. Antibody binding was visualized using standard streptavidin-biotin peroxidase (SAB) methodology (Dako Ltd., Denmark) with di-aminobenzidine $0.5 \mathrm{mg} / \mathrm{ml}$ (DAB Sigma Ltd., UK) acting as the chromogen. Endogenous peroxidase activity was inhibited in the normal manner $\left(3 \% \mathrm{v} / \mathrm{v} \mathrm{H}_{2} \mathrm{O}_{2}\right.$ in distilled water for $5 \mathrm{~min}$ ). All specific antibody IHC reactivity was found to be dependent upon antigen retrieval protocols. All tissue sections were counterstained with $5 \%$ v/v Harris haematoxylin, dehydrated, cleared in xylene and mounted in DPX mountant. Positive (known antigen expressing tissue sections) and negative controls (no-primary antibody) were included in each batch of slides.

RNA binding: mobility shift assays and inhibition of nucleic acid binding. The ability of antibodies, to inhibit the binding of MDM2 to RNA was determined employing modified gel mobility shift assays. Standard binding assays were performed in accordance with methodology established in our laboratories to study both RNA and DNA binding of capacities of a recombinant MDM2 protein expressed and extensively purified after transfection and induction of an FL-h-MDM2 pET15b construct transfected into E. coli BL21. Briefly, MDM2 and control random riboprobes were produced as follows: a DNA oligonucleotide termed T7EBSp6 corresponding to the previously reported RNA/ MDM2 consensus binding sequence but flanked by $\mathrm{T} 7$ and SP6 promoter sites, was synthesized having the following sequence: (5'-TGTAATACGACTCACTATAGGGCGA ACCGCCGGAUCUUAAAUAAACUCCUGGUCUA GTATTCTATAGTGTCACCTAAAT-3'). The sequence was amplified via PCR utilising sense (Sn) T7 and antisense (Asn) Sp6 primers facilitating production of a dsDNA intermediate which was resolved on and extracted from a 5\% T/2.6\% C polyacrylamide TBE gel and ultimately further purified using a Qia-Quick (Quiagen, UK) DNA purification column (manufacturer's protocols). This dsDNA as well as a randomized cassette of 50 nucleo-tides (N50) RNA (control) were then in vitro transcribed employing the Ampliscribe SP6 in vitro transcription system (Promega Ltd., USA). Transcription from the SP6 promoter progressed in the presence of $\alpha^{-}{ }^{32} \mathrm{P}-\mathrm{UTP}$ (400 $\mathrm{Cimmol}^{-1}$ ) as well as unlabelled CTP, GTP and ATP. The ribo-probes so created were treated with DNAse I for $30 \mathrm{~min}$ at $37^{\circ} \mathrm{C}$ and then phenol extracted with the final RNA being precipitated with $5.2 \mathrm{M}$ ammonium acetate $\mathrm{pH} 4.8$ in ethanol in the presence of carrier linear acrylamide. Probes were pelleted by centrifugation $(14,000$ $\mathrm{x}$ g), washed with $70 \%$ ethanol, re-pelleted and re-suspended in 10-20 ml of DEPC water. Finally, the concentration and specific activity of the probes were determined prior to use.

In binding assays FL-MDM2 protein $(0.5 \mu \mathrm{g})$ was incubated together with $0.2-0.5 \mathrm{ng}(100,000 \mathrm{cpm})$ of either of the above labeled ribo-probes in a reaction volumes of $15 \mu 1$. Effectively probe was diluted in binding buffer $(0.2 \mathrm{mM}$ EDTA, $2 \mathrm{mM} \mathrm{MgCl}_{2}, 0.5 \mathrm{mM}$ DTT and $0.5 \mathrm{mM}$ PMSF in PBS), heated to $70^{\circ} \mathrm{C}$, and allowed to slowly cool to $30^{\circ} \mathrm{C}$. After addition of the protein, binding reactions were allowed to proceed for $15 \mathrm{~min}$ at $23^{\circ} \mathrm{C}, 20 \mathrm{U}$ of RNAse T1 was added to each reaction, incubated for $5 \mathrm{~min}$ at room temperature, $50 \mu \mathrm{g}$ (1 $\mu$ l) of calcium heparin was then added and the reactions allowed to proceed for 5 min prior to transferring to ice. RNA was then covalently linked to the protein by UV irradiating the complexes for $720 \mathrm{sec}$ in a standard Stratagene-Stratolinker. The reaction mixtures were then incubated at $37^{\circ} \mathrm{C}$ with $20 \mathrm{U}$ of RNAse for $10 \mathrm{~min}$. At this point normal binding reactions were taken up in dissociation buffer and resolved on polyacrylamide gels together with probe alone to visualize any RNase resistant secondary structure. Labeled products and their relative mobilities were visualized by Phosphor-imaging. Super-shift assays (utilizing MDM2 specific antibodies after complex formation) were further used to confirm the MDM2 specificity of the binding activity inferred by the mobility shifts observed in the RNA binding assays. Antibody-RNA binding inhibition experiments were performed using a modification of the standard RNA binding assay protocol in which serial dilutions of immuno-affinity purified antibody were pre-incubated with FL-recombinant MDM2 protein (500 ng) prior to reaction with $0.20-5 \mathrm{ng}(100,000 \mathrm{cpm})$ of the consensus nucleic acid MDM2 binding sequence probe, retaining reaction volumes of $15 \mu \mathrm{l}$. Reduction of binding was analyzed by EMSA and the shifts achieved were compared with or without antibody pre-treatment.

In vitro expression of MDM2 isoforms. cDNA clones corresponding to FL-MDM2 and alternately spliced forms of MDM2 (16) and a luciferase control were employed in coupled in vitro transcription/translation reactions using the Promega TNT rabbit reticulocyte lysate system following manufacturer's instructions. In vitro translated proteins were radio labeled with ${ }^{35} \mathrm{~S}$ (Amersham Ltd., UK) by allowing translation to progress at $30^{\circ} \mathrm{C}$ for $1.5 \mathrm{~h}$ (luciferase control) or $2 \mathrm{~h}$ (MDM2 samples) in the presence of free aminoacids including ${ }^{35} \mathrm{~S}$-methionine. Confirmation of successful translation and labeling was achieved using a combination of SDS PAGE (4-20\% Tris-glycine polyacrylamide gels) and Phospho-Image analysis (Molecular Dynamics). The amount of protein translated was quantified using SYPRO-Orange ${ }^{\circledR}$ (Genetic Research Instrumentation, UK).

In vivo expression of MDM2 isoforms. Constructs incorporating the differing $M D M 2$ isoforms were prepared 


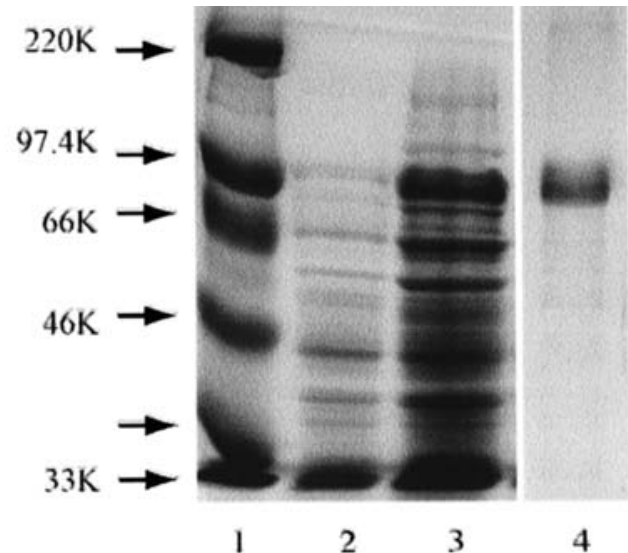

Figure 1. Expression and purification of recombinant FL-MDM2: coomassie blue stained gel showing: lane 1, molecular weight markers; lane 2, crude pre-IPTG induction sample; lane 3 , crude post-IPTG induction sample; lane 4, $\mathrm{Ni}^{2+} \mathrm{His}$ column purified MDM2. Pooled wash and eluate fractions containing the specific FL MDM2-protein.

utilizing the ecdysone-inducible expression system developed and marketed by Invitrogen. This system was adopted as previous work had shown that the pCR3 vector system into which various MDM2 isoforms had been introduced by Sigalas et al (16) initially elicited low in vivo expression of MDM2 protein products. This Invitrogen $\mathrm{pVgRxR}$ high expression system allowed cDNA clones corresponding to each of the MDM2 isoforms to be placed under expression control of a hybrid ecdysone response element (E/GRE) inducible by ponasterone A, a synthetic analogue of ecdysone. These constructs in which expression is ultimately under he control of the Drosophila HSP promoter were then transfected into NIH3T3 cells establishing lines able to inducibly express significant amounts of RNA from each of the sub-cloned major MDM2 isoforms studied. Cultures of these cells harboring the transfected constructs were lysed as described previously and the products resolved on $4-20 \%$ Tris-glycine acrylamide gradient gels and the resolved proteins transferred to BA85 nitrocellulose membrane. The membranes were then probed with purified antibody from the MDM2-specific antibodies and antigen/antibody complexes so formed were visualized using ECL techniques cited above.

Immunoprecipitation of translated MDM2 proteins with antiMDM2 antibodies. In order to identify the MDM2 isoforms reactive with F2-2 (IgG1) and F4-14 (IgG1) antibodies, a series of immunoprecipitation reactions were performed utilising the ${ }^{35} \mathrm{~S}$-methionine in vitro translated products described above. Immunoprecipitation of the translated MDM2 proteins was achieved employing each anti-MDM2 antibody successively. This was achieved using an immunomagnetic separation technique employing a range of Fc $\gamma$ chain specific secondary antibody-linked Dynabeads M-280 (Dynal Ltd., UK). These beads were reacted with in-house 'anti-MDM2' antibodies $\left(1.5 \mathrm{mg} / 10^{7}\right.$ beads linked at $4^{\circ} \mathrm{C}$ for $20 \mathrm{~h}$ ), washed and the resulting MDM2-antibody-coated Dynabeads separated and then reacted with in vitro translation reaction mixes $\left(2 \mathrm{~h}\right.$ at $\left.4^{\circ} \mathrm{C}\right)$. Antibody-antigen complexes were collected on the MPC [magnetic particle concentrator (Dynal)], washed and re-suspended in $45 \mu 1$ aliquots of SDS sample buffer which was boiled depleted of beads in the MPC and run (5 $\mu \mathrm{l})$ on SDS-PAGE Tris-glycine gradient gels (4-20\%). Immunoprecipitated MDM2 isoforms were all run alongside crude in vitro translation reactions to allow direct comparisons to be made. Resolved radiolabeled products were visualized by Phosphor-imaging.

\section{Results}

Production and purification of FL recombinant MDM2 protein for generation of anti-MDM2 antibodies. Amplification of the coding sequence of $M D M 2$ from $\mathrm{CaCo}_{2}$ cells and the subsequent cloning and sub-cloning schedules described, successfully created a construct, which allowed expression of FL MDM2 in E. coli. BL21 cells. This protein was successfully purified by $\mathrm{Ni}^{2+}$ column chromatography (Fig. 1) and this final product, migrating at approximately $97.4 \mathrm{kDa}$ (FL-protein plus vector encoded poly-histidine tag and thrombin cleavage sequences), served both as immunogen, in the described immunization schedules, and capture antigen in successive rounds of hybridoma screening via ELISA.

Production of monoclonal antibodies. The adopted immunization schedules successfully elicited serum antibody responses within the 10 -week schedule described, as indicated by circulating anti-MDM2 specific serum antibody titres in excess of $1 / 100,000$. A number of NS-1 primed splenocyte fusions were performed successfully, each giving plating efficiencies in excess of 95\%. Approximately 200 hybridoma lines which actively secreted the antibody that bound to homologous MDM2 protein, were selected for further study. After limiting dilution, a number of group specific hybridoma clones were grown to provide stocks of antibody purified by Protein A/G column chromatography. These could be divided into a number of sub-groups based upon the results of ELISA, Western blotting and immunohistochemical analyses of reactivity and further analyzed for their ability to interfere with nucleic acid binding. The following sections describe the characterization of two clones, F4-14 and F2-2 and which displayed inhibitory activity in MDM2 nucleic acid binding assays.

Immunohistochemistry. Immunohistochemistry (IHC) was carried out using both the F2-2 and F4-14 anti MDM2 monoclonal antibodies in parallel with a number of well-established commercially available MDM2 antibodies (Fig. 2). This comparison was performed using sections of primary breast carcinoma tissue, known to over-express MDM2 protein. Negative control (i.e. no primary antibody control and negative tissue controls) sections were included in this comparison. Established commercially available MDM2 specific antibodies (IF2 Oncogene Sciences and 9E3 NovocastraLaboratories) produced a typical nuclear staining pattern on breast carcinoma sections known to over-express the MDM2 protein (Fig. 2c and d). The new antibodies F2-2 and F4-14 also produced comparable patterns of nuclear immunostaining on sections of the same tumor tissue (Fig. 2a and b). Each produced both an overall intense nuclear stain in many tumor nuclei but also displayed a defined perinuclear 

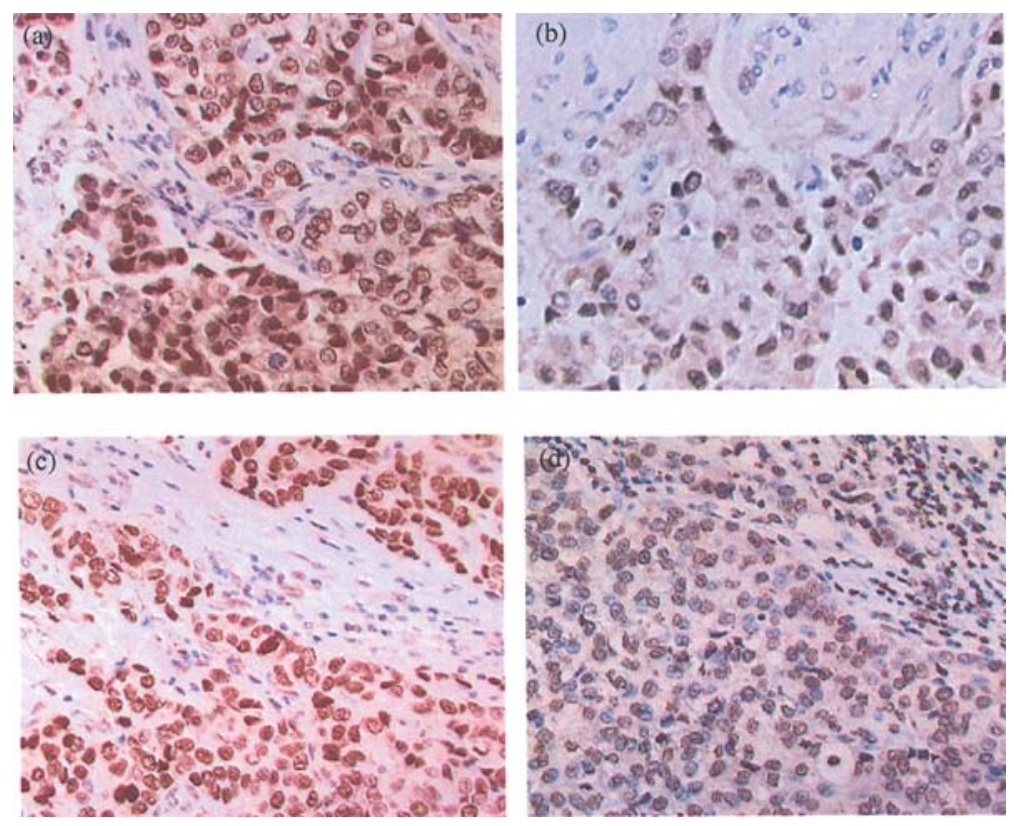

Figure 2. Immunohistochemical staining of sections of primary breast carcinoma sections with MDM2 specific monoclonal antibodies. (a) Clone F4-14; (b), clone F2-2; (c) clone IF-2; (d) clone 9E3.
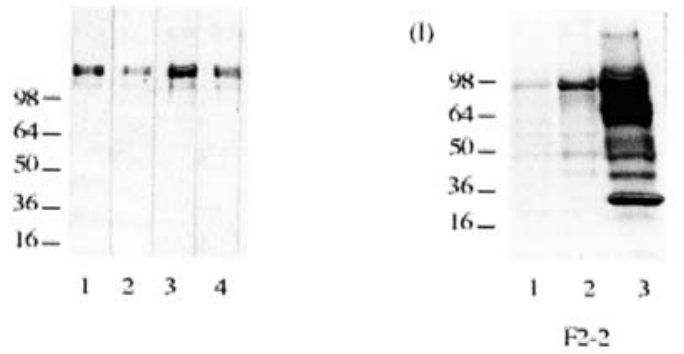

(II)

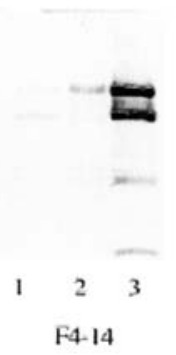

Figure 3. Reactivity of antibodies with MDM2 in bacterial recombinant poly-histidine-tagged MDM2 protein preparations and tumour cell lysates. (a) Recombinant FL-MDM2 (poly-His): lane 1, monoclonal mouse antibody clone F2-2 (1:2500); lane 2, monoclonal mouse antibody clone F4-14 (1:2500); lane 3, monoclonal mouse antibody clone IF-2 (1:100); lane 4, polyclonal rabbit antibody SMP14 (1:100). (b) Tumour cell lysates and in vitro transcribed protein: (I), F2-2 antibody: lane 1, A2780 ovarian epithelial adenocarcinoma cell line WT P53 and MDM2; lane 2, SJSA-1 sarcoma (Femur) WT P53, MDM2 gene amplified; lane 3, in vitro coupled transcription translation FL-MDM2 cDNA. (II), F4-14 antibody: lane 1, A2780 ovarian epithelial adenocarcinoma cell line WT P53 and MDM2; lane 2, SJSA-1 sarcoma (Femur) WT P53, MDM2 gene amplified; lane 3, in vitro coupled transcription translation FL-MDM2 cDNA.

staining pattern often accompanied by diffuse particulate staining of a significant proportion of tumor cell nuclei.

Reactivity of F2-2 (IgG1) and F4-14 (IgG1) monoclonal antibodies with recombinant MDM2 or MDM2 occurring in tumor cell lysates in Western blotting. Fig. 3a illustrates reaction of F2-2 and F4-14 antibodies with FL poly-histidinetagged MDM2 protein purified from E. coli BL21 lysates with equal protein loading. Similarly, Fig. 3b shows specific reactivity and the ability of both antibodies to detect differences in expression of 'native' MDM2 in lysates of A2780 (p53+/p53+) cells an ovarian epithelial adenocarcinoma, and SJSA-1 (p53+/+) a sarcoma (femur) cell line known to be amplified for the MDM2 gene. However, reactivity with a number of additional bands was also evident. The estimated molecular weights of these additional bands corresponded to the projected weights of a number of the different protein isoforms encoded by the MDM2 gene. However, given the observations reported in the following section regarding the electrophoretic mobility of MDM2-protein isoforms translated in vitro in the TNT-cell free system it is difficult to define the identity of these bands on the basis of predicted molecular weight alone.

Reactivity of F2-2 (IgG1) and F4-14 (IgG1) monoclonal antibodies with MDM2 protein isoforms analyzed by Western blotting and immunoprecipitation. In order to more closely assess the ability of F2-2 and F4-14 antibodies to recognize both FL- and specific protein isoforms of MDM2, a series of Western blotting and immunoprecipitation experiments were performed employing pCR3.1 plasmid expression constructs encoding FL-MDM2 or each of the alternately spliced MDM2 variants $(A-G)$ created previously in our laboratories. Initial 
(a)

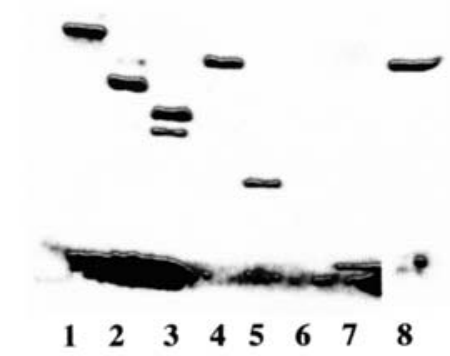

(b)

\begin{tabular}{|c|c|c|c|c|}
\hline Variant & $\begin{array}{c}\text { Transcript } \\
\text { Size }\end{array}$ & $\begin{array}{c}\text { Amino-Acid Num- } \\
\text { ber }\end{array}$ & $\begin{array}{c}\text { Predicted Protein } \\
\text { Mr }\end{array}$ & $\begin{array}{c}\text { Apparent Mr } \\
\text { PAGE }\end{array}$ \\
\hline FL & 1473 & 491 & 56000 & 90000 \\
\hline a & 810 & 270 & 30793 & 75000 \\
\hline b & 654 & 218 & 24862 & 48000 \\
\hline C & 963 & 321 & 36610 & 85000 \\
\hline d & 396 & 132 & 15054 & 30000 \\
\hline e & 306 & 102 & 11663 & 16000 \\
\hline$f$ & 261 & 87 & 9922 & 14000 \\
\hline
\end{tabular}

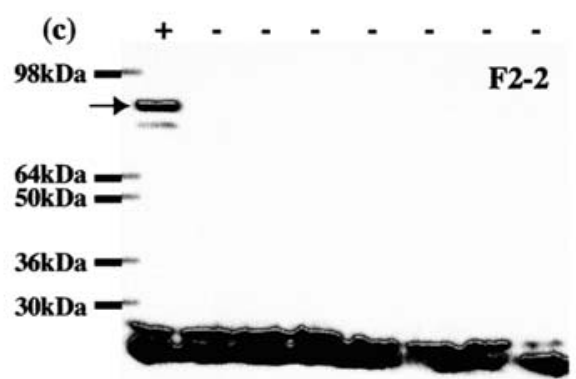

(d) $+++++-\cdot$

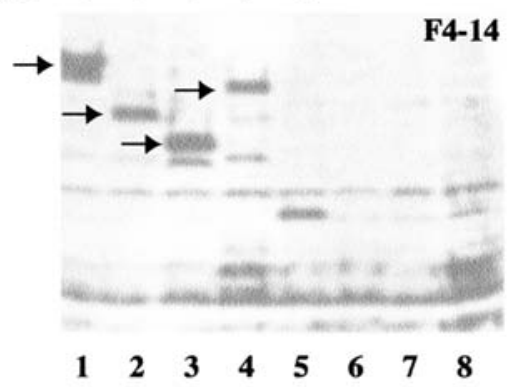

(e)

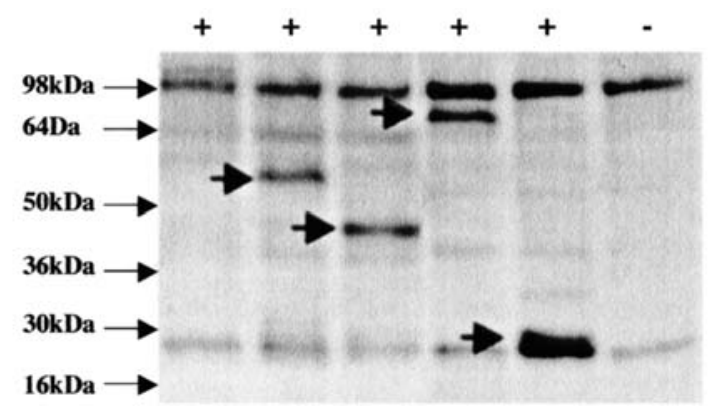

Figure 4. (a) SDS-PAGE showing in vitro transcription/translation products for MDM2 variants. Lane 1, FL-MDM2; lane 2, MDM2 variant a; lane 3, MDM2 variant b; lane 4, MDM2 variant c; lane 5, MDM2 variant d; lane 6, MDM2 variant e; lane 7, MDM2 variant f; lane 8; control (Luciferase). (b) Comparison of predicted and apparent Mr of MDM2 protein isoforms in SDS-PAGE gels (c and d) Western blots showing recognition of FL-MDM2 and variants by monoclonal antibodies F2-2 and F4-14 after in vitro translation of products from pCR constructs: lane 1, FL-MDM2; lane 2, MDM2 variant a; lane 3, MDM2 variant b; lane 4, MDM2 variant c; lane 5, MDM2 variant d; lane 6, MDM2 variant e; lane 7, MDM2 variant f; lane 8, control (Luciferase). (e) Western blot of antibody F4-14/MDM2 isoform immunoprecipitates formed from lysates of NIH3T3 cells transfected with pVgRxR-MDM2 constructs: lane 1, FL-MDM2; lane 2, MDM2 variant 1a; lane 3, MDM2 variant b; lane 4, MDM2 variant $\mathrm{c}$; lane 5, MDM2 variant d; lane 6, MDM2 variant e.

results showed that RNAs from all eight MDM2-isoform encoding pCR3.1 constructs were successfully transcribed and subsequently translated using the TNT rabbit reticulocyte in vitro translation system Fig. 4a. However, different degrees of efficiency of transcription and or translation were apparent, this being evident by the varying amount of protein product produced. This was particularly true of the MDM2-E construct that in this system yielded a relatively small amount of protein in comparison with the other constructs. In the manner in which the projected Mr of FL-MDM2 and its corresponding estimated mass defined by PAGE have proven disparate, so differences were observed between the projected and estimated masses of each of the in vitro translated products. Following in vitro translation, clone F2-2 was found to react with only the full-length protein isoform of MDM2 failing to recognize protein isoforms A to $\mathrm{G}$ (Fig. 4c). In contrast, monoclonal antibody F4-14 was found to bind the fulllength product as well as isoforms A, B, C and D (Fig. 4d). However, it was not possible to detect any reaction of this antibody with either isoforms $\mathrm{E}$ or F. Immunoprecipitation of radiolabeled in vitro translated products produced results comparable to those of blotting experiments (data not shown). In vitro transfection studies with $\mathrm{pVgRxR}$ high expression constructs, and combined immunoprecipitation and immunoblotting, further confirmed the reactivity profiles of the two antibodies (clone F4-14 reactivity shown in Fig. 4e). Utilising this high expression system confirmed that all isoforms studied produced significant amounts of RNA detectable by Northern blotting (data not shown), including constructs encoding isoforms E. Thus the observation that apparent failure of reactivity of each antibody with defined isoforms was not likely to be associated with a lack of the protein product, which had remained a possibility as the efficiency of expression from the pCR3.1 vector system is considerably less than that of the $\mathrm{pVgRxR}$ system.

Inhibition of MDM2-specific nucleic acid binding by monoclonal antibodies F2-2 and F4-14. Radiolabelled consensus RNA binding sequence (T7EBSP6) consistently bound to recombinant FL-MDM2 protein in EMSA allowing 
(a)

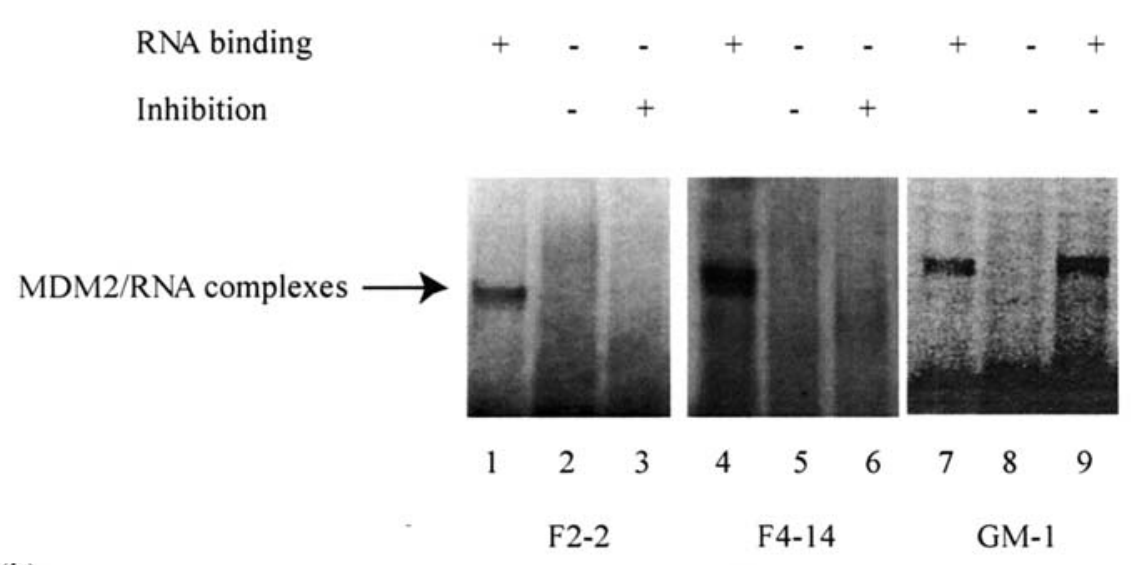

(b)

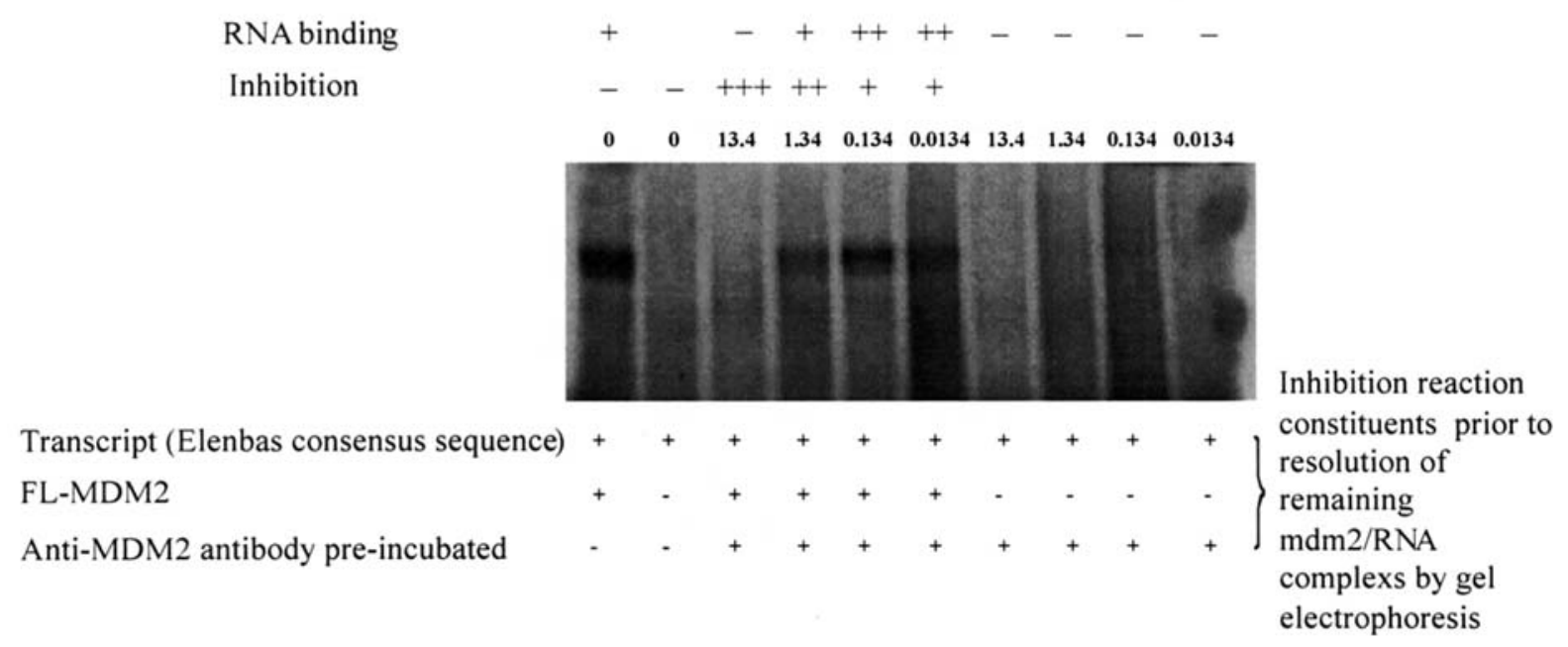

Figure 5. (a) Mobility shift assay showing in vitro transcribed ${ }^{32} \mathrm{P}-\mathrm{UTP}$ labelled RNA reacted with: lanes 1, 4 and 7, FL-MDM2 protein (0.5 mg); lanes 2, 5 and 8, Tris- $\mathrm{HCl}$ alone (no protein); lane 3 , FL-MDM2 protein $(0.5 \mathrm{mg})$ pre-incubated with F2-2 monoclonal antibody; lane 6 , FL-MDM2 protein (0.5 mg) preincubated with F4-14 monoclonal antibody; lane 9, FL-MDM2 protein (0.5 mg) pre-incubated with GM-1 (anti-cyclin D1) monoclonal antibody. (b) Mobility shift assay as in (a) but pre-reacting the protein with varying concentrations of F4-14 antibody (concentration of antibody in mg shown above each lane): lane 1, no antibody control; lane 2, no MDM2 protein control; lanes 3, 4, 5 and 6 antibody inhibition assays; lanes 7, 8, 9 and 10 antibody controls.

visualization of the MDM2/RNA complex (Fig. 5a, tracks, 4 and 7). In order to investigate the abilities of F2-2 and F4-14 monoclonal antibodies to inhibit nucleic acid binding a series of modified EMSA tests were performed. Initial mobility shift assays employing recombinant poly-histidine tagged FL-MDM2 protein confirmed the ability of the bacterially expressed protein to bind an oligo-ribonucleotide corresponding to the T7EBSp6 consensus sequence defined by Elenbaas et al (12). Pre-incubation of high concentrations of either the F4-14 or F2-2 antibodies with recombinant FLMDM2 protein prior to reaction with the T7EBSp6 RNA sequence inhibited the capacity of FL-MDM2 to bind this consensus RNA sequence (Fig. 5a, tracks 3 and 6). High concentrations of a non-related antibody (M-Ab-GM1 an anti-cyclin D1 antibody) failed to inhibit RNA binding confirming the inhibitory effect on RNA binding was F4-14/ F2-2 dependent. Incubation of either antibody with preformed MDM2:RNA complexes, did not prevent antibody binding, nor did antibody binding induce dissociation of complexes but rather produced a classic super-shift due to the creation of a tri-molecular complex (data not shown). FL-
MDM2 RNA binding was significantly inhibited by reducing concentrations of both antibodies, although the degree of inhibition achieved with F4-14 was greater than that achieved with F2-2. Inhibition of binding was evidently dependent on antibody concentration and reduced with serially increasing dilutions of antibody (Fig. 5b - titration of F4-14). RNA binding was re-established and comparable with control binding when levels of F2-2 pre-incubated with protein fell below $1.8 \mu \mathrm{g} /$ assay. In comparison F4-14 completely inhibited binding at high concentrations $(13.4 \mu \mathrm{g} /$ assay), however a significant reduction of binding effected by F4-14 relative to the control (Fig. 5b, track 1) remained apparent when pre-incubation antibody concentrations were reduced to $13 \mathrm{ng} /$ assay (Fig. 5b, track 6). In the presence of antibody and T7EBSp6 alone no non-specific binding was apparent (Fig. 5b, tracks 7 to 10).

Additional data in an allied series of experiments had previously demonstrated an ability of FL-MDM2 protein to bind to DNA although a conventional consensus binding sequence was not identified. However, whilst each antibody inhibited RNA binding to FL-MDM2 protein, to differing 

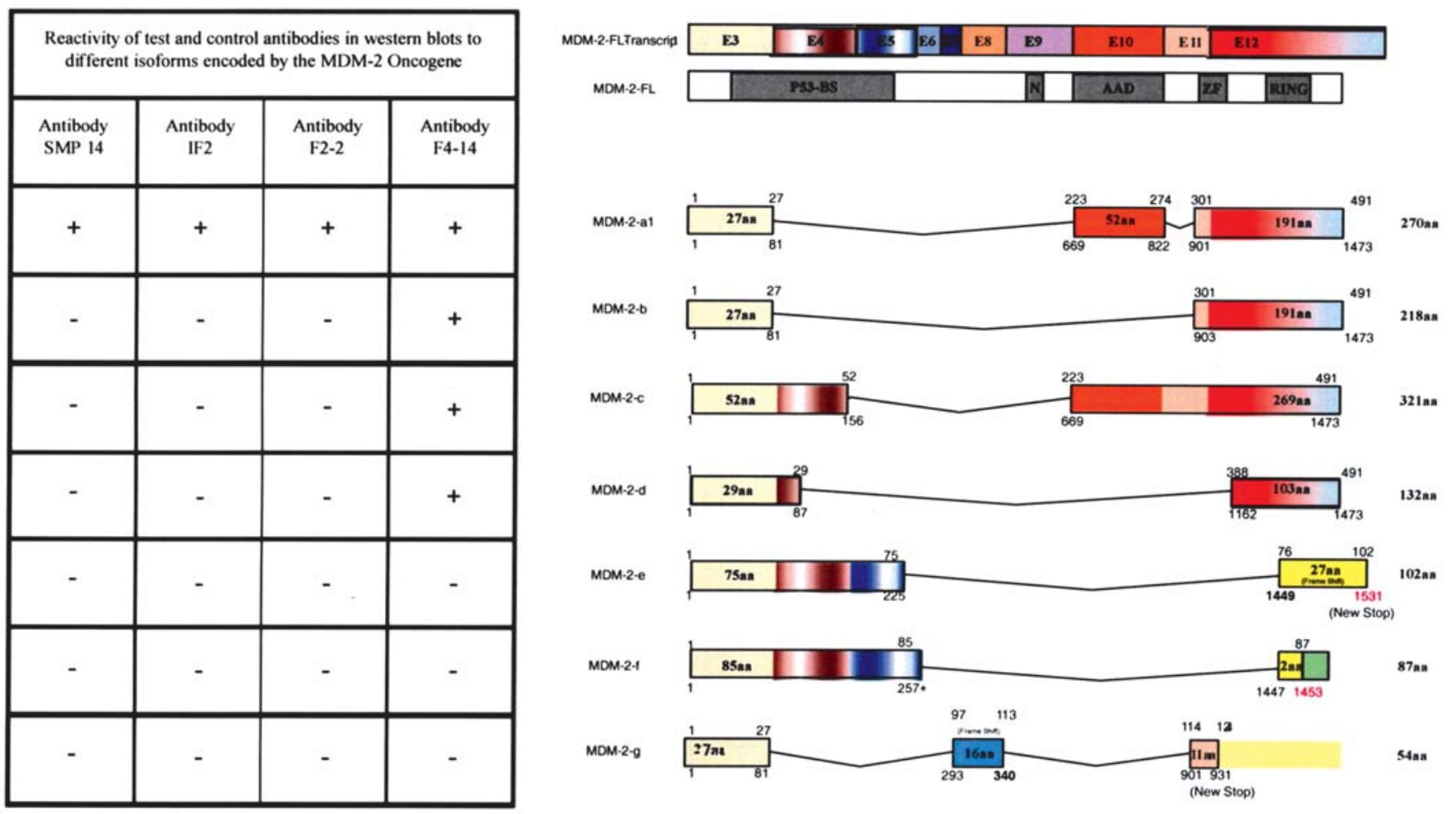

Figure 6. Reactivity of test and control monoclonal antibodies in Western blots in relation to the protein isoforms encoded by the defined alternately spliced transcripts of the MDM2 oncogene.

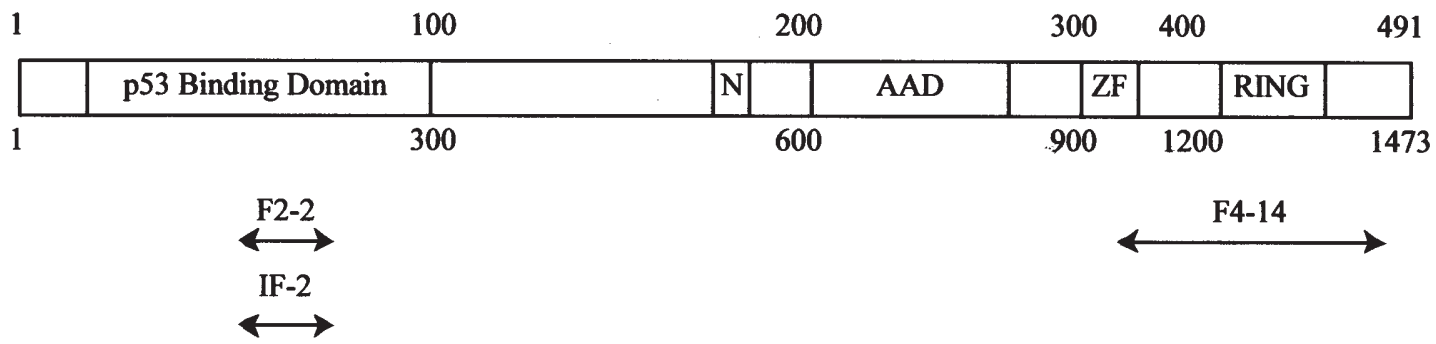

Figure 7. Epitope map of commercial and new monoclonal MDM2 antibodies against full-length MDM2. Also illustrated are the positions of the p53 binding domain, nuclear localisation signal $(\mathrm{N})$, acidic activation domain (AAD), zinc finger (ZF) and RING finger domains relative to the putative binding sites of each of the three antibodies detailed.

extents (F4-14 >> F2-2), neither F4-14 nor F2-2 inhibited DNA binding when pre-incubated with FL-MDM2 recombinant protein prior to complexing with reactive DNA clones. This suggested that different regions of MDM2 are involved in DNA and RNA binding. Based on Western blotting and immunoprecipitation of in vitro translated or in vivo expressed isoforms of MDM2 (summarized in Fig. 6), we have putatively assigned the regions of F4-14 reactivity to the area encompassing both the C proximal RING finger domain (Fig. 7).

\section{Discussion}

Full-length poly-histidine-tagged recombinant MDM2 protein was successfully expressed in and purified from $E$. coli. This purified protein engendered significant serum antibody responses when used to immunize Balb/c laboratory mice in accordance with our conventional schedules. Fusion of primed splenocytes from these immunized mice with NS-1 cells then allowed the successful establishment of a series of hybridoma lines and ultimately a panel of such clones secreting MDM2 protein specific monoclonal antibodies. Two clones F2-2 and F4-14 were selected from this pool for further study. Both selected antibodies proved able to react with FL-MDM2 in ELISA, immunohistochemistry and Western blotting. In SAB-immunohistochemistry both these particular new antibodies produced patterns of immunostaining essentially comparable with a number of commercially available antibodies although a number of differences were apparent. When breast carcinoma tissue sections which over expressed MDM2 were stained via IHC, the new antibodies also displayed a more defined peri-nuclear pattern of staining accompanying a diffuse particulate staining of a significant portion of tumor cell nuclei. This indicated that although 
novel antibodies and the commercial antibodies reacted with MDM2 products the localization of the products detected in IHC might perhaps differ somewhat. The MDM2 protein specificity of antibodies F4-14 and F2-2 is confirmed by Western blots of protein lysates derived from tumor cell lines known to express varying amounts of MDM2 (Fig. 3b). This demonstrated clearly that both selected antibodies were specific, and potentially reactive with multiple MDM2 protein isoforms and able to detect differences in the expression of MDM2 in these cell lines in comparison to actin controls.

In order to define the protein products of MDM2, which F2-2 and F4-14 recognize, Western blotting and immunoprecipitation experiments were performed. Immunoblotting and immunoprecipitation experiments with in vitro translated MDM2-pCR constructs revealed that F2-2 strongly reacted in Western blots with only FL-MDM2 (Figs. 4c and 6), This supported the observation of strong reactivity of this antibody with FL-MDM2 protein in lysates of SJSA-1 (p53 wt $\left.{ }^{+}\right)$ femoral carcinoma cells in which the MDM2 gene is known to be amplified (Fig. 3b). In comparison in vitro translation, and in vivo $\mathrm{pVgRxR}$ expression experiments indicated that antibody F4-14 reacted strongly on Western blots with FLMDM2 as well as variants a, b, c, d (Figs. 4d and 6). Control commercially produced monoclonal antibodies SMP14 and IF-2 (raised against synthetic $\mathrm{N}$ proximal peptide: amino acids 154-167 and an $\mathrm{N}$ proximal recombinant protein a.a. 25-168) reacted strongly only with FL-MDM2 in Western blotting (data not shown). F2-2, similar to these commercial antibodies, recognizes only the full length molecule and yet is able to modulate binding of the Elenbaas et al RNA consensus sequence which is thought to bind at some point within the RING Finger binding domain (12). In contrast, F414 which recognizes MDM2 protein products A to D would appear to react with residues within the carboxy-terminal portion of the molecule as the only region common to all four of these proteins locates between amino acids 389 and 491, the C-proximal portion of MDM2 D after the splice site, and the region encompassing the RING finger (Fig. 6). However, given the current data, we cannot preclude the possibility that other contact residues recognized by this antibody may lie outside this region if the antibody recognition site were to form a discontinuous epitope. The ability of this antibody to bind in this region, however, would account for its greater RNA inhibitory binding capacity than antibody F2-2.

Further experiments are now in progress to determine more accurately and specifically the precise contact residues constituting the epitopes recognized by each antibody. Preliminary experiments utilizing the Novagen-Nova TopepScreen-1b based epitope mapping system have confirmed the presence of the binding site of commercial antibodies SMP14 within the immunizing peptide and indicating that of IF-2 to map between a.a. 140 and 154. The same technique, which is dependent upon immunoblotting indicated that antibody F2-2 reacted within 211-235. Thus, binding of this antibody may exert some inhibitory effect on RNA binding possibly as a result of steric hindrance, as the recognition site of the antibody may become juxtaposed to the binding site during protein folding rather than direct specific occupation of the RNA binding site. Unfortunately, we were unable to define the site recognized by antibody F4-14 applying the same methodology suggesting that the antibody may react with a discontinuous epitope present in FL-MDM2 as well as isoforms A to D.

While we were successfully able to demonstrate inhibition of RNA binding with these antibodies, we were unable to inhibit the novel MDM2 DNA binding cited earlier in this report. This may reflect the possibility that the observed DNA binding does not occur through the RING domain and therefore implies a different mechanism for DNA binding.

Considerable research has been done on the interaction of MDM2 and p53, which interact with one another in a "noncontinuous' manner involving multiple minimal structure motifs in the $\mathrm{N}$ terminal transactivation domain of p53 (a.a. 18-26, 40-45 and 49-54) and an $\mathrm{N}$ terminal pocket domain of MDM2 (located within the N proximal 100 a.a.) (24). These interactions initiate the processes that facilitate the maintenance of the homeostatic feedback loop between these molecules, which are central to the cells response to genotoxic and stress related injury. For some time the sole function of MDM2 was considered as the autocrine regulator of p53 ensuring its E3 dependent degradation and restoration of balance. However, it is now becoming evident that this process of interaction and the activity of MDM2 is far from that of solely a ubiqutin ligase. Currently, in excess of 40 MDM2 RNA transcripts have been described as well as a variety of protein isoforms (17), the primary forms of which have been proposed to be full length $\mathrm{p} 90^{\mathrm{MDM} 2}$ and $\mathrm{p} 75^{\mathrm{MDM} 2}$ proteins, the transcripts of which are derived preferentially from the P2/P1 and P1 promoters respectively (25). The physiological significance of expression of different MDM2 protein isoforms still remains the subject of substantial speculation, evidence supports an oncogenic role of FL protein although shorter transcripts have been attributed greater oncogenic potential and advanced disease (16). However, conversely, additional evidence suggests that protein isoforms with the RING finger domain intact may bind to FL-MDM2 thereby reducing p53 binding facilitating p53 accumulation and promoting associated anti-proliferative and pro-apoptotic effects. Thus, the role of MDM2 protein isoforms remains somewhat enigmatic and dependent upon the tumor type and the primary importance or even level of redundancy of the p53/MDM2 feedback system in any given tumor lineage. Although our current knowledge of MDM2 activities is biased towards p53 dependent functions of MDM2 more is becoming evident of the complexity of the targets of MDM2 interactions as well as p53-independent functions. Newly acknowledged functions of these protagonists include the recognition of p53/MDM2 and TSG101/MDM2 feedback loops and the differential roles played by different MDM2 isoforms, as well as the interaction of MDM2 with targets including, chromatin, various p53 dependent and independent (human telomerase receptor) response elements and promoter respectively, as well as oncogenic isoforms of related molecules such as the MDMX11 transcript (26-28). The significance of the role of interaction of MDM2 with a specific RNA consensus binding sequence remains relatively unexplored. MDM2 was shown to complex with the ribosomal L5 protein, and the L5/5S ribonucleosome, although this binding was dependent upon the acidic 
activation domain of and independent of RING finger dependent binding to the defined consensus RNA sequence reproduced in this study. This led to the suggestion that MDM2 may be involved in translational regulation within the cell in addition to the p53 dependent and independent transcriptional regulation activities that it possesses. The ability of monoclonal antibody F4-14 to avidly block this binding supports the suggestion that contact residues lie within the RNG finger close to or able to interfere with the reaction of the consensus RNA with amino acid residue G446 that was shown to be critical for consensus sequence binding. It is hoped that this antibody may now further allow us to modulate MDM2 RNA binding in vivo and explore the resultant physiological effects induced by influencing the proposed MDM2 imposed translational control. In the longer term this may offer the potential for exploitation of novel intervention or therapy.

In conclusion, we have produced two antibodies which successfully inhibit MDM2 RNA binding, but not DNA binding, and are reactive with FL-MDM2 protein as well as a number of protein isoforms of MDM2, in one or more of a variety of assays including ELISA, immunohistochemistry, Western blotting and immunoprecipitation. From the results described here we have putatively mapped the recognition sites of both F2-2 and F4-14 antibodies (Fig. 7), although further experiments are in progress to accurately and specifically map the epitope sites of these new MDM2 monoclonal antibodies. The ability of these antibodies particularly F4-14 to inhibit and thereby 'regulate' RNA binding raises the possibility of modulating this function and enabling future investigation of the physiological significance of this activity. In the longer term this may offer the potential for identification and development of useful and therapeutic targets.

\section{Acknowledgements}

We would like to acknowledge the support of the UK Cancer Research Campaign for sponsorship of this work.

\section{References}

1. Farmer G, Bargonetti J, Zhu H, Friedman P, Prywes R and Prives C: Wild-type p53 activates transcription in vitro. Nature 358: 83-86, 1992

2. Barak Y, Juven T, Haffner R and Oren M: $m d m 2$ expression is induced by wild-type p53 activity. EMBO J 12: 461-468, 1993.

3. Oliner JD, Kinzler KW, Meltzer PS, George DL and Vogelstein B: Amplification of a gene encoding a p53-associated protein in human sarcomas. Nature 358: 80-83, 1992.

4. Chen J, Marechal V and Levine AJ: Mapping of the p53 and mdm2 interaction domains. Mol Cell Biol 13: 4107-4114, 1993.

5. Boddy MN, Freemont PS and Borden KL: The p53-associated protein MDM2 contains a newly characterized zinc-binding domain called the RING finger. Trends Biochem Sci 19: 198-199, 1994.

6. Wu XW and Bayle JH: The P53 Mdm2 autoregulatory feedback loop. Genes Dev 7: 1126-1132, 1993.
7. Picksley SM and Lane DP: The p53-mdm2 autoregulatory feedback loop: a paradigm for the regulation of growth control by p53? Bioessays 15: 689-690 1993.

8. Fakharzadeh SS, Trusko SP and George DL: Tumorigenic potential associated with enhanced expression of a gene that is amplified in a mouse tumor cell line. EMBO J 10: 1565-1569, 1991

9. Li M, Brooks CL, Wu-Baer F, Chen D, Baer R and Gu W: Mono-versus polyubiquitination: differential control of p53 fate by Mdm2. Science 302: 1972-1975, 2003.

10. Minsky N and Oren M: The RING domain of Mdm2 mediates histone ubiquitylation and transcriptional repression. Mol Cell 16: 631-639, 2004.

11. Levav-Cohen $Y$, Haupt $\mathrm{S}$ and Haupt $\mathrm{Y}: \mathrm{Mdm} 2$ in growth signaling and cancer. Growth Factors 23: 183-192, 2005.

12. Elenbaas B, Dobbelstein M, Roth J, Shenk T and Levine AJ: The MDM2 oncoprotein binds specifically to RNA through its RING finger domain. Mol Med 2: 439-451, 1996.

13. Leach FS, Tokino T, Meltzer P, et al: p53 mutation and MDM2 amplification in human soft tissue sarcomas. Cancer Res 53: 2231-2234, 1993.

14. Momand J, Jung D, Wilczynski S and Niland J: The MDM2 gene amplification database. Nucleic Acids Res 26: 3453-3459, 1998.

15. Landers JE, Haines DS, Strauss JF III and George Dl: Enhanced translation: a novel mechanism of mdm2 oncogene over-expression identified in human tumor cells. Oncogene 9: 2745-2750, 1994.

16. Sigalas I, Calvert AH, Anderson JJ, Neal DE and Lunec J: Alternatively spliced mdm 2 transcripts with loss of $\mathrm{p} 53$ binding domain sequences: transforming ability and frequent detection in human cancer. Nat Med 2: 912-917, 1996.

17. Bartel F, Taubert $\mathrm{H}$ and Harris LC: Alternative and aberrant splicing of MDM2 mRNA in human cancer. Cancer Cell 2: 9-15, 2002.

18. Onel K and Cordon-Cardo C: MDM2 and prognosis. Mol Cancer Res 2: 1-8, 2004.

19. Wurl P, Meye A, Berger D, et al: Prognostic relevance of Cterminal Mdm2 detection is enhanced by p53 positivity in soft tissue sarcomas. Diagn Mol Pathol 6: 249-254, 1997.

20. Gorgoulis VG, Zoumpourlis V, Rassidakis GZ, Karameris A, Rassidakis AN, Spandidos DA and Kittas C: A molecular and immunohistochemical study of the MDM2 protein isoforms and p53 gene product in bronchogenic carcinoma. J Pathol 180: 129-137, 1996.

21. Anderson JJ and Lunec J: The prognostic significance and interactions of p53 in human cancer. European Society of Oncology Scientific Updates 1: 77-93, 1996.

22. Cordon-Cardo C, Latres E, Drobnjak M, et al: Molecular abnormalities of mdm2 and p53 genes in adult soft tissue sarcomas. Cancer Res 54: 794-799, 1994.

23. Lodge AJ, Anderson JJ, Ng SW, et al: Expression of topoisomerase IIIalpha in normal and neoplastic tissues determined by immunohistochemistry using a novel monoclonal antibody. Br J Cancer 83: 498-505, 2000.

24. Chi SW, Lee SH, Kim DH, et al: Structural details on mdm2p53 interaction. J Biol Chem 280: 38795-38802, 2005.

25. Cheng TH and Cohen SN: Human MDM2 isoforms translated differentially on constitutive versus p53-regulated transcripts have distinct functions in the p53/MDM2 and TSG101/MDM2 feedback control loops. Mol Cell Biol 27: 111-119, 2007.

26. White DE, Talbott KE, Arva NC and Bargonetti J: Mouse double minute 2 associates with chromatin in the presence of p53 and is released to facilitate activation of transcription. Cancer Res 66: 3463-3470, 2006.

27. Zhao J, Bilsland A, Jackson K and Keith WN: MDM2 negatively regulates the human telomerase RNA gene promoter. BMC Cancer 5: 6, 2005.

28. Giglio S, Mancini F, Gentiletti F, et al: Identification of an aberrantly spliced form of HDMX in human tumors: a new mechanism for HDM2 stabilization. Cancer Res 65: 9687-9694, 2005. 